\title{
INDETERMINATENESS OF ELECTRICAL CHARGE
}

\author{
By Chester Snow
}

\section{ABSTRACT}

It is shown that if every electric charge in the world be given a charge of true magnetism, their ratio being a constant, the change could not be detected. If the electric and magnetic charges of an electron are, respectively, $\epsilon_{1}$ and $\lambda_{1}$, then we have measured only $\sqrt{\epsilon_{1}+\lambda_{1}{ }_{1}}$. The algebraic signs of $\epsilon_{1}$ and $\lambda_{1}$ are arbitrary, but the ratio $\epsilon_{1} / \lambda_{1}$ must be the same as for a proton and is physically indeterminate. The proposition may be regarded as a one-parameter transformation of the electromagnetic field components (in the same system of coordinates), which leaves the electromagnetic energy tensor unchanged. This indeterminateness is rooted in the electromagnetic scheme and, therefore, pervades the interior of all electrons which have been constructed on that scheme. A place is found for it in the world geometry of Eddington.

In spite of the perfect parallelism in the definitions of electric charge and charge of true magnetism, there is no evidence for the existence of the latter. There are no currents of magnetism, and no isolated magnetic charge of either sign has ever been found analogous to the discovery of electrons. With the explanation of magnetic polarization in terms of Amperean electric currents or rotating electric charges, the last use for the concept of magnetism disappeared. The success of the electron theory and the electromagnetic theory of light have led to the conclusion that all matter is constructed of electric charges.

One of the strongest inner confirmations of the theory of relativity is the naturalness with which the Maxwell-Lorentz field equations lead not only to the invariance of electric charge but also to its conservation. The vanishing of the divergence of the four-vector representing the density of the electric current charge merely expresses the fact (in Galilean coordinates) that any appearance of electricity at a point may be accounted for in a natural manner by its coming to that point along some path in space. Electric charge has a permanent existence. A velocity may be assigned to it. Perhaps no better definition of substance may be given, and the electrical theory of matter proclaims it the one substance.

It may be pointed out, however, that electricity serves as a measure of this one substance only by a tacit convention which is implicit in the whole development of the electron theory. Let us suppose that $69183^{\circ}-27$ 
some, being wearied with the view of this too-electrical universe, should impart to each electrical charge a magnetic one proportional to its strength. It will be shown presently that no one on earth could know the difference. This wedding of electricity and magnetism would, indeed, be a fruitless union. So many electromagnetic fictions intervene between our contacts with reality that it is possible to add this new one without fear of detection. It is undoubtedly pedantic but equally irrefutable to say that Millikan did not measure the electric charge $\epsilon_{1}$ of an electron (if it has one) nor the magnetic charge $\lambda_{1}$ (if it has one), but only $\sqrt{\epsilon_{1}{ }_{1}+\lambda^{2}}$, which it possesses by definition.

Assuming for the present that we are concerned with a region where the special theory of relativity is valid, we believe that for any Galilean coordinates there are two vectors, $e$ and $h$, which "define the electromagnetic state of the world." This full-sounding phrase enables us to avoid the self-consciousness experienced by a reference to ether. It is usually believed that we are able to evaluate these vectors in a unique manner as long as we remain in the same system of coordinates. The electric and magnetic densities $\rho_{1}$ and $\rho_{2}$, for all our christening and substantializing of them, are simply defined as the divergence of $e$ and $h$, respectively. From a consideration of the geometry of the four-dimensional world, we believe that there are certain relations between $e, h, \rho_{1}, \rho_{2}$, and their velocities $u_{1}$ and $u_{2}$, which express properties of the world which are equally fundamental with those expressed by the law of gravitation.

In the present coordinate systems these relations are

$$
\begin{aligned}
\operatorname{curl} h-\dot{e} & =\rho_{1} u_{1} \\
\operatorname{div} e & =\rho_{1} \\
\operatorname{curl} e+\dot{h} & =-\rho_{2} u_{2} \\
\operatorname{div} h & =\rho_{2}
\end{aligned}
$$

We are, of course, considering these equations in the microscopic sense, according to which all currents are convection currents. The present theory places $\rho_{2}=0$. It is not inconsistent with the theory of relativity to assume that neither $\rho_{1}$ nor $\rho_{2}$ is zero, and we are attempting to find out whether it is also consistent with experience. Relativity demands that both electric charge and magnetic charge in a given volume be invariant.

The condition of compatibility of the equation (1) is

and that for (2) is

$$
\dot{\rho}_{1}+\operatorname{div}\left(\rho_{1} u_{1}\right)=0
$$

$$
\dot{\rho}_{2}+\operatorname{div}\left(\rho_{2} u_{2}\right)=0
$$


In the past we have read into equation (3) the conservation of something and infer that it may be useful in describing the permanent existence of the one substance with which we are acquainted, and which we have called electricity. As we do not know of any other substance, we have read no such meaning into (4) but have been able to account for experience quite successfully by assuming that $\rho_{2}$ is everywhere zero. The equation (3) has for us a profound significance. The equation (4) is reduced to a trivial identity. Since my purpose is to call attention to a harmless indeterminateness in our electric theory, I prefer to recognize in (4) another aspect of the permanent existence of the selfsame substance to which (3) refers. However, it is important to remember that all of the equations just written connect ideal quantities, and no observations or measurements could have reference to them alone. If we knew the electromagnetic state of the world-that is, the vectors $e$ and $h$ we could find the densities $\rho_{1}$ and $\rho_{2}$ by taking their divergence; and, conversely, if we know $\rho_{1}$ and $\rho_{2}$ and their velocity at all points we could find $e$ and $h$. But we have no direct cognizance of either.

The contact with physics (dyrramics) is made by the attempt to satisfy experimental observations by assuming that a particle having a volume $d v$ and charges $\rho_{1} d v$ and $\rho_{2} d v$ when at rest in a given field would experience an acceleration $\frac{d u}{d t}$, given by

$$
\left(m_{0} \frac{d u}{d t}\right)_{u=0}=\left\{\rho_{1} e+\rho_{2} h\right\} d v
$$

where $m_{0}$ is a constant, the invariant mass. The special theory of relativity then shows that its motion, in general, must satisfy

$$
\frac{d}{d t}\left(\frac{m_{0}}{\sqrt{1-u^{2}}} u\right)=d v\left(\rho_{1}\{e+[u, h]\}+\rho_{2}\{h-[u, e]\}\right)
$$

so that the electromagnetic force per unit volume is

$$
f=\rho_{1}\{e+[u, h]\}+\rho_{2}\{h-[u, e]\}
$$

The expression of electrical theory is in this equation, the explanation of the terms here used being given by (1) and (2). According to the view here set forth that the one substance always has electric charge and magnetic charge in a fixed ratio, it is evident that the velocity of this substance is $u=u_{1}=u_{2}$ and

$$
\rho_{2}=\rho_{1} \tan \alpha
$$

where $a$ is a universal constant as yet undetermined.

Let us now introduce the new density

$$
\rho \equiv \rho_{1} \cos a+\rho_{2} \sin \alpha
$$


This, together with equation (6), shows that

$$
\rho_{1}=\rho \cos \alpha \text { and } \rho_{2}=\rho \sin \alpha
$$

If we define two new vectors by

$$
\left.\begin{array}{l}
E \equiv e \cos a+h \sin a \\
H \equiv-e \sin a+h \cos a
\end{array}\right\}
$$

we find from (1) and (2), placing $u=u_{1}=u_{2}$, that

$$
\begin{aligned}
\operatorname{curl} H-\dot{E} & =\rho u \\
\operatorname{div} E & =\rho \\
\text { curl } E+\dot{H} & =0 \\
\operatorname{div} H & =0
\end{aligned}
$$

If we now compute the electromagnetic force by (5), we find

$$
f=\rho\{E+[u, H]\}
$$

Thus in equations (10), (11), and (12) we fall back upon the complete foundations of the present-day theory of electricity, the electrical theory of matter and of light. It appears that $a$ is not so much a universal constant as an indeterminate parameter. If we place $a= \pm n \pi$, we get a purely electrical theory of matter; if $\alpha= \pm(2 n+1)$ $\frac{\pi}{2}$, a purely magnetic one which is just as simple. The mutual influence of two electrons which are moving in any possible manner, or of two protons, or of an electron and a proton, the laws of induced currents or electromagnetic force between currents, the energy density, the flow of energy or Poynting vector, the Maxwell stresses, the microscopic equations with their picture of electric and magnetic polarization-in short, the physical world-is obviously unaltered by the new interpretation which replaces the negative electric charge of an electron by $\pm \sqrt{\epsilon_{1}{ }_{1}+\lambda^{8}{ }_{1}}$, where $\epsilon_{1}$ and $\lambda_{1}$ are its new electric and magnetic ones, each capable of having either algebraic sign, provided that the positive electrical charge on a proton be replaced by $\mp \sqrt{\epsilon_{2}^{2}+\lambda_{2}^{2}}$, where the two upper or two lower signs of these two radicals must, of course, be associated together, and the four algebraic quantities must satisfy the relation

$$
\frac{\epsilon_{1}}{\lambda_{1}}=\frac{\epsilon_{2}}{\lambda_{2}}
$$

This will have little interest for pragmatic philosophers. Evidently only certain combinations of the vectors which determine the state of the world can ever be known to us. Other combinations may forever slip through our fingers. 
It may be that by insisting upon the possibility of transforming with impunity a purely electrical universe into what may properly be called an electromagnetic one, and by thereby accusing the entire electrical scheme of physical indeterminateness, we are somewhat overbearing. And not so much because of the inconsequential nature of this transformation as on account of the fact that we are not recognizing its origin and limitations. May we go inside the electron with this transformation? Assuredly, wherever its creators have carried inside the electromagnetic scheme of equations (1) and (2) as well as the electromagnetic force of equation (5).

If in the electrical electrons of Lorentz or Abraham one redistributes the electric into electric and magnetic charge in any arbitrary ratio, the non-Maxwellian binding force (hidden discreetly in the background) will never know the difference. The electromagnetic mass of these electrons will obviously be unaffected.

This does not answer the question. The moral might be drawn that the electromagnetic scheme should not have been carried inside the electron where even the simple concept of velocity may be inapplicable. It is quite possible that the boundary of the electron may be the boundary of this thesis among others. Nevertheless, if electrons and protons are not mathematical point charges, we may say that the roots of this indeterminateness do not lie in the atomicity of charges, but simply in the electromagnetic scheme (1) and (2), from which the definitions of electricity and magnetism are derived, together with the electromagnetic force (5). Wherever this scheme is carried the indeterminateness follows like a shadow.

By casting the argument here presented into tensor form and denoting by $J_{2}{ }^{\mu}$ the four-vector representing magnetic current and charge, the assumption $J_{2}{ }^{\mu}=J_{1}{ }^{\mu} \tan \alpha$ is readily seen to be admitted into the general theory of relativity. There is a difference, however, between being admitted and in finding a reserved seat. When we turn to the world geometry of Eddington, ${ }^{1}$ which places the electromagnetic potentials on a level equally fundamental with that of the gravitational potentials, one might naturally expect to find there some evidence for the purely electrical theory of matter which would rule out the magnetic aspect or, at least, discourage it. On the contrary, I find there a place which seems to be waiting for it.

Eddington, from a consideration of the general relation structure of the world, brings up for world-building material the fundamental in tensor ${ }^{*} G_{\mu \nu}$ which involves among other things the RiemannChristoffel tensor $G_{\mu \nu}$ and two vectors, $k_{\mu}$ and $\lambda_{\mu}$. Identifying the symmetrical part of ${ }^{*} G_{\mu \nu}$ with the gravitational potentials $g_{\mu \nu}$, he obtains the gauging equation for the natural gauge of the world, which in empty space reduces to the law of gravitation. The transition

1 Eddington, The Mathemstical Theory of Relativity, 1923, Cambridge Press, pp. 213-228. 
from the geometrical description to the physical description is made by inquiring first what properties the physical tensor possesses and then seeking among the geometrical tensors on hand one which possesses these properties by virtue of mathematical identities. Thus he convincingly identifies the complete energy tensor with $G_{\mu}{ }^{\nu}-\frac{1}{2} g_{\mu}{ }^{\nu}(G-2 \lambda)$, because both have the striking property of conservation expressed by the vanishing of their contracted covariant derivative, and arrives at the law of gravitation for space which is not empty. The electromagnetic potentials are then placed on an equal footing with the gravitational ones by identifying the vector $k_{\mu}$ with electromagnetic potential, so that the electromagnetic tensor is its four-dimensional curl; that is,

$$
f_{\mu \nu}=\frac{\partial k_{\mu}}{\partial x^{\nu}}-\frac{\partial k_{\nu}}{\partial x^{\mu}}
$$

Its contracted covariant derivative furnishes us with a four-vector,

$$
\left(f^{\mu \nu}\right)_{\nu}=J_{1}^{\mu}
$$

which is identified with electric current-charge density because, due to the antisymmetry of $f^{\mu \nu}$, it has the property of conservation

$$
\left(J_{1}{ }^{\mu}\right)_{\mu}=0
$$

From the fact that the antisymmetrio tensor $f_{\mu \nu}$ is of the special form (13), it follows that the following tensor equation is satisfied identically,

$$
\frac{\partial f_{\nu \sigma}}{\partial x^{\mu}}+\frac{\partial f_{\sigma_{\mu}}}{\partial x^{\nu}}+\frac{\partial f_{\mu \nu}}{\partial x^{\sigma}}=0
$$

The conclusion is almost irresistible that in equation (15) we have the expression of the permanent existence of our one substance which we have called electricity. In Galilean coordinates it must reduce to our equation (3) and fuv must take the form

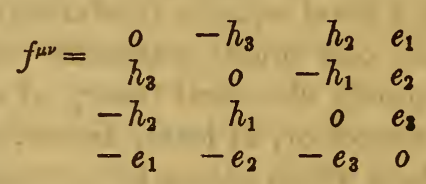

The equation (13) reduces to three-vector equations

$$
\begin{aligned}
& e=-\dot{a}-\Delta \phi \\
& h=\operatorname{curl} a
\end{aligned}
$$

where $a$ is the ordinary vector potential and $\phi$ the scalar potential, while (14) reduces to the first set of field equations (1) and (16) to the second set (2), in which the magnetic density $\rho_{2}$ must be placed equal to zero-a requirement also indicated by (18), which requires that $\operatorname{div} h=0$. 
Consequently, a world geometry which, like Weyl's, provides us with a single four-vector $k_{\mu}$ as a potential out of which all the field equations flow is a one-substance theory from the start, either purely electrical or purely magnetic. This is not true, however, if we are furnished with a tensor $f_{\mu \nu}$ which is merely antisymmetric, for then the second member of (16) is not identically zero and a magnetic current is indicated. The assumption that $f_{\mu \nu}$ is of the form (13) is another way of placing $\rho_{2}=0$.

Neither is the magnetic aspect of our one-substance ruled out if we are furnished, as in Eddington's geometry, with a second vector $\lambda_{\mu}$, which defines another antisymmetrical tensor

$$
h_{\mu \nu}=\frac{\partial \lambda_{\mu}}{\partial x^{\nu}}-\frac{\partial \lambda_{\nu}}{\partial x^{\mu}}
$$

which defines a second current-charge vector

$$
\left(h^{\mu \nu}\right)_{\nu}=-J_{2}^{\mu}
$$

which has the same remarkable property of conservation

$$
\left(J_{2}{ }^{\mu}\right)_{\mu}=0
$$

There is also the tensor equation

$$
\frac{\partial h_{\nu \sigma}}{\partial x^{\mu}}+\frac{\partial h_{\sigma \mu}}{\partial x^{\nu}}+\frac{\partial h_{\mu \nu}}{\partial x^{\sigma}}=0
$$

In these four sets of equations we have another one-substance theory. If the conclusion from (15) was irresistible, that from $\left(15^{\prime}\right)$ should be equally so. As we know of only one substance and do not care to fly in the face of facts by peopling the universe with another substance which has never been found, let us recognize in $\left(15^{\prime}\right)$ the magnetic aspect of the permanence of the same substance. (If there are other aspects, we may meet the same type of equation again.) In Galilean coordinates it must reduce to our equation (4) and $h^{\mu \nu}$ must reduce to

$$
h_{\mu \nu}=\begin{array}{cccc}
0 & -e_{3} & e_{2} & -h_{1} \\
e_{3} & 0 & -e_{1} & -h_{2} \\
-e_{2} & e_{1} & 0 & -h_{3} \\
h_{1} & h_{2} & h_{3} & 0
\end{array}
$$

The equation $\left(13^{\prime}\right)$ then reduces to the three-vector set of equations

$$
\begin{gathered}
e=\operatorname{curl} b \\
h=\dot{b}+\Delta \psi
\end{gathered}
$$

where $b$ is a vector and $\psi$ a scalar potential, while (14') reduces to our field equations (2) and (16') reduces to (1), in which $\rho_{1}$ must be 
placed equal to zero, as required also by $\left(18^{\prime}\right)$, since $\operatorname{div} e$ must be zero.

On comparing (18) and $\left(18^{\prime}\right)$ (which require $e$ on the one hand to be the curl of a vector and on the other hand forbid it to be the curl of any vector), we might suppose that we are here confronted with two incompatible one-substance theories and that we are compelled to reject one of them. That, in fact, is what Eddington does. He does not identify $\lambda_{\mu}$ with an electromagnetic potential because, having already identified it with $k_{\mu}$ - that is, $f_{\mu \nu}$ - "that would leave $f_{\mu \nu}$ with nothing to do." But he leaves $\lambda_{\mu}$ with nothing to do, which, indeed, should not be criticized. For although, as I shall show, we may assimilate these two apparently contradictory schemes into our one electrical theory in an infinite variety of ways, we shall always leave something with nothing to do. Certain combinations of the world tensors will elude us.

We must not regard the two tensors $f_{\mu \nu}$ and $h_{\mu \nu}$ as antagonistic, one affirming what the other denies. Rather let us think of $f_{\mu \nu}$ as referring only to the electrical charge of the one substance. That is why its $h$ is the curl of $a$. The tensor $h_{\mu \nu}$ refers only to the magnetic charge of this substance, and that is why its $e$ is the curl of $b$. If we now introduce our peculiar contention that the one substance has associated with it both electric and magnetic charge in some fixed but undetermined ratio, then

$$
J_{2}^{\mu}=J_{1}^{\mu} \tan \alpha
$$

It may well be that neither $J_{1^{\mu}}$ nor $J_{2}{ }^{\mu}$ can ever be known to us separately, but only some combination, such as

$$
J^{\mu} \equiv J_{1}{ }^{\mu} \cos \alpha+J_{2}{ }^{\mu} \sin \alpha \text { or }\left\{\begin{array}{l}
J_{1^{\mu}}=J^{\mu} \cos \alpha \\
J_{2}{ }^{\mu}=J^{\mu} \sin a
\end{array}\right.
$$

Consequently $f_{\mu \nu}$ and $h_{\mu \nu}$ may not be capable of evaluation, but only the combination

We then find that

$$
F_{\mu \nu} \equiv f_{\mu \nu} \cos \alpha-h_{\mu \nu} \sin \alpha
$$

$$
\begin{gathered}
\left(F^{\mu \nu}\right)_{\nu}=J^{\mu} \\
\left(J^{\mu}\right)_{\mu}=0 \\
\frac{\delta F_{\nu \sigma}}{\delta x^{\mu}}+\frac{\delta F_{\sigma \mu}}{\delta x^{\nu}}+\frac{\delta F_{\mu \nu}}{\delta x^{\sigma}}=0
\end{gathered}
$$

This last equation shows that $F_{\mu \nu}$ is the four-dimensional curl of some four potential $A_{\mu}$, so that

$$
F_{\mu \nu}=\frac{\partial A_{\mu}}{\partial x^{\nu}}-\frac{\partial A_{\nu}}{\partial x^{\mu}} \text { or }\left\{\begin{array}{l}
E=-A-\Delta \Phi \\
H=\operatorname{curl} A
\end{array}\right.
$$

In the last four equations we recognize the present electrical theory. 
Evidently the single tensor $F_{\mu \nu}$ extracts from the two world tensors $f_{\mu \nu}$ and $h_{\mu \nu}$ all that can have significance for us. The indeterminateness we are discussing is thus seen to find a place in Eddington's world geometry. Indeed, it would not be difficult to believe that he had some such indeterminateness in mind for, having constructed all that he set out to build and finding on hand a superfluity of worldbuilding material (such as the vector $\lambda_{\mu}$ ), he disposes of this dead lumber with the illuminating remarks that "We are only aware of a selection of things which exist (in an extended meaning of the word), the selection being determined by the nature of the apparatus available for exploring nature." Also, "it is quite unnecessary to specialize the possible relation structure of the world in such a way that the useless variables have the fixed value zero; that loses sight of the interesting result that the world will go on just the same if they are not zero."

In conclusion, it may be noted that the vector $A_{\mu}$ may be increased by any covariant vector of type $\frac{\partial V}{\partial x^{\mu}}$, where $V$ is a function of the four coordinates, without affecting the values of $F_{\mu \nu}$. This is usually interpreted by the statement that it is not the potentials $A_{\mu}$ but the field components $F_{\mu \nu}$ which determine for us the electromagnetic state of the world.

This statement must be modified. Even the field components $F_{\mu \nu}$ do not uniquely determine for us the electromagnecic state, not even in one Galilean system, for the equations (6) and (9) may be regarded as a one-parameter transformation (with arbitrary parameter a) of the electromagnetic scheme, which leaves the world unaltered as far as we are able to discriminate. This is a shifting of all the charges in this electrical universe into electric and magnetic in any arbitrarily fixed ratio and is quite different from any transformation of coordinates such as is contemplated in the general theory of relativity.

Viewing equation (9) as a transformation from one pair of vectors, $e$ and $h$, to another pair, $E$ and $H$, in the same Galilean system, it is readily found that the two "invariants" of the electromagnetic field $\frac{h^{2}-e^{2}}{2}$ and $(e, h)$ are not invariant for this change but are connected by the relations

$$
\begin{aligned}
& \frac{H^{2}-E^{2}}{2}=\frac{h^{2}-e^{2}}{2} \cos 2 a-(e, h) \sin 2 a \\
& (E, H)=\frac{h^{2}-e^{2}}{2} \sin 2 a+(e, h) \cos 2 a
\end{aligned}
$$


The motive underlying this transformation was to confer invariance upon the electromagnetic force. This it does in a rather simple manner, for it will be found that the energy density, $\frac{e^{2}+h^{2}}{2}$, the electromagnetic momentum density or Poynting vector $[e, h]$, and each component of the Maxwell stresses remain unaltered. In short, each component of the electromagnetic energy tensor remains unaffected, which is another way of showing that the "change" will escape detection. The resolution of charge into electric and magnetic constituents is analogous to the resolution of a plane vector into two rectangular components and may be made in an infinite number of ways.

In the interpretation of electrical experiments there need be no such unanimity as is generally supposed among the inhabitants of the same Galilean system of coordinates. Each could choose his own $a$ and, therefore, his own electromagnetic vectors $e$ and $h$, and each could see his own real Faraday tubes sweeping through space. Having arrived at such a condition, no discussion is wholly useless which serves to emphasize how large a part of our physical thought is concerned with imaginary constructions. ${ }^{2}$

Washington, August 24, 1927.

2 In a forthcoming paper gravitation is viewed as the (imaginary) magnetic aspect of all matter. The indeterminateness of charge here set forth persists in that view and is taken as an indication that space-time is part of a six-dimensional world possessing a simple geometry, the electric axis being timelike, the imaginary magnetic being spacelike, the orientation of these axes being physically indeterminate. The charge is a hypervector and, since all mass has in general both fleld-producing aspects, any. particle moves on a geodetic line of this six-dimensional space. 